\title{
Pharmaceutical Devices for Oral Cavity-based Local and Systemic Drug Delivery

\author{
${ }^{1}$ Department of Food Science and Technology, Chung-Ang University, Ansung, Korea \\ ${ }^{2}$ Division of Pharmaceutics, College of Pharmacy, Chung-Ang University, Seoul, Korea \\ ${ }^{3}$ School of Pharmacy, Sungkyunkwan University, Suwon, Korea \\ ${ }^{4}$ Bioavailability Control Laboratory, College of Pharmacy, Kangwon National University, Chuncheon, Korea
} \\ Gyiae Yun ${ }^{1,2}$, Sung-Up Choi ${ }^{2}$, Ki-Hwan Park', Yun-Seok Rhee ${ }^{3}$, Beom-Jin Lee ${ }^{4}$ and Jaehwi Lee ${ }^{2 \dagger}$
}

(Received August 24, $2010 \cdot$ Revised October 6, 2010 • Accepted October 7, 2010)

\begin{abstract}
Pharmaceutical technology has primarily focused on the development of the best dosage forms depending on the route of administration. The design of dosage forms is greatly influenced by the route of administration. Due to a variety of advantages such as avoidance of first-pass effect, abundant blood supply and easy access to the absorption site, the oral cavity has frequently been selected as a site for drug delivery. Since the oral cavity is relatively unique from the anatomical and physiological viewpoint, one should always consider these conditions when designing the drug delivery systems for the oral cavity. In this regard, the current review paper was prepared to summarize the essential features of the drug delivery systems utilized in the oral cavity, along with the introduction of various dosage forms developed to date.
\end{abstract}

Key words - Oral cavity, Buccal, Bioadhesive, Mucoadhesive, Drug delivery system, Dosage forms

A number of buccal delivery systems are being investigated and have been used for drug absorption via the oral mucosa. These include aqueous solutions (e.g. mouth wash, a dose $\leq$ $25 \mathrm{~mL}$ ), conventional buccal and sublingual tablets, liquidfilled capsules, adhesive tablets, adhesive gels, adhesive patches, chewing gums, metered-dose aerosols and devices attached to the teeth or implanted in the tooth enamel (Merkle et al., 1990). The selection of dosage form primarily relies on the therapeutic intention, such as rapid or prolonged effect and systemic or local therapeutic effect, as well as the physicochemical properties of the drug molecules. It would be additionally important to increase the patient acceptability of the delivery system i.e. ease of use and avoidance of discomfort. Karsenty et al. (1994) have evaluated clinically the patient acceptance of a transmucosal patch (a matrix of polyacrylic acid and polyisobutylene with adhesive impermeable backing layer) with regard to comfort, irritation and feeling when presented and removed. They reported that it is important to demonstrate patient acceptance if the delivery system is to gain a wide range of applicability for a number of therapies. It is also important that the additives used to formulate oral mucosal dosage forms do not stimulate salivary secretion as this may cause the loss of a large fraction of the active ingredient prior to absorption. Rathbone et al. (1994) have reported that the

†Corresponding Author :

Tel : +82-2-820-5606, E-mail : jaehwi@cau.ac.kr

DOI : $10.4333 /$ KPS.2010.40.S.113 size, shape and the thickness of the delivery system should be considered to maximise patient compliance. Anders and Merkle (1989) have proposed the appropriate dimensions of dosage forms for buccal use. For an inflexible conventional tablet, $5-8 \mathrm{~mm}$ in diameter is suitable for comfort and the ability of the patient to constantly detect its presence. In the case of a flexible patch, the maximum size would be $10-15 \mathrm{~cm}^{2}$. Sublingual and gingival patches are rather smaller (no more than $1-3 \mathrm{~cm}^{2}$ ) than those for buccal mucosa. Ellipsoidal patches have been shown to be the most suitable shape to be placed centrally on the buccal mucosa. As mentioned above, since the dosage forms used for drug delivery in the oral cavity requires special features it would be essential to summarize their characteristics from the literature.

\section{Oral Cavity as a Site for Drug Delivery}

\section{Anatomical properties of the oral cavity}

The oral cavity has been selected extensively as a potential site for local and systemic delivery of therapeutic agents. Local therapy has treated conditions such as gingivitis, oral candidiasis, oral lesions, dental caries and xerostoma using delivery systems which have included mouthwashes, chewing gums, bioadhesive tablets, gels and patches (Nantwi et al., 1997). Since the successful delivery of glyceryl trinitrate across sublingual mucosa for the treatment of angina pectoris, the oral cavity has been investigated extensively for systemic delivery (de Vries et al., 1991). Although the buccal and sub- 
lingual mucosae are the primary sites for the drug absorption, all mucosal surfaces in the oral cavity are discussed here.

The following terms are defined for reasons of clarity and used throughout (Rathbone et al., 1994).

- Oral cavity - the area of the mouth delineated by the lips, cheeks, hard palate, soft palate and floor of the mouth.

- Oral mucosa - the membranes that line the oral cavity which include the sublingual, buccal mucosa, the gums (gingivae), the palatal mucosa and the labial mucosa.

- Buccal mucosa - the membrane inside the mouth that lines the cheek.

The sublingual mucosa is considered as the membrane on the ventral surface of the tongue and on the floor of the mouth.

The oral cavity is lined with stratified squamous epithelium below which lies the basement membrane supported by a lamina propria. The lamina propria is composed of collagen fibres, a supporting layer of connective tissue, blood vessels and smooth muscle (Gandhi and Robinson, 1994). Masticatory mucosa covers the gingiva and hard palate and consists of a keratinised epithelium (i.e. cornified surface) that resembles the epidermis of the skin in terms of the pattern of maturation. On the other hand, the lining mucosa covers the remaining part of the oral mucosa except for the dorsal surface of the tongue (specialised mucosa). This lining mucosa does not possess a keratinised layer of the epithelium and is more permeable than a keratinised region or skin. The surface of the lining mucosa is less resistant to mechanical abrasion but is flexible and extensible to allow mobility of the lining region.

\section{Drug delivery in the oral cavity}

It has long been established that drugs can be absorbed via the oral mucosa since glyceryl trinitrate was delivered through the sublingual mucosa (de Vries et al., 1991). However, understanding and identifying the drug absorption process and mechanism via the oral mucosa at the cellular and molecular level still remain challenging due to the complexity caused by the physico-chemical features of the cell and tissue and permeant, and the interaction between them. The epithelial cell membrane is, in fact, lipophilic and is based on the fluid mosaic model representing biological membranes composed of a fluid-state lipid bilayer embedded with globular integral proteins. On the other hand, the intercellular region is comparatively hydrophilic despite the fact that membrane-coating granules secret a number of lipophilic components.

Exogenous substances are transported across the buccal epithelial mucosa by means of simple (passive) diffusion (Shojaei and Li, 1997), carrier mediated diffusion (Utoguchi et al., 1996) and presumably active transport or other specialised mechanisms such as endocytosis. Paracellular and transcellular routes are two transport pathways for passive diffusion. The permeation of polar molecules such as peptide-based therapeutic agents often occurs by the paracellular route. Since the paracellular pathway is situated at the aqueous extracellular region, some structural features such as molecular size, charge and hydrophilicity are involved in the absorption process as well as the area of the intercellular space and its tortuosity. The steady-state flux $\left(J_{\mathrm{P}}\right)$ model for hydrophilic drugs through the paracellular route was proposed by Zhang and Robinson (1996):

$$
J_{P}=\frac{D_{P} \cdot \varepsilon}{h_{P}} \cdot C_{D}
$$

where $\varepsilon$ is the area fraction of the paracellular route, $h_{p}$ is the length of the paracellular pathway, $D_{p}$ is the diffusion coefficient in the intercellular spaces and $C_{D}$ is the concentration of drug in the donor chamber.

In contrast, lipophilic compounds are transported across lipophilic biological membranes (transcellular) and the steadystate flux $\left(J_{\mathrm{T}}\right)$ is given by equation 1.2 (Zhang and Robinson, 1996):

$$
J_{T}=\frac{(1-\varepsilon) \cdot D_{T} \cdot K_{P}}{h_{T}} \cdot C_{D}
$$

where $K_{P}$ is the partition coefficient between the lipophilic phase (cell membrane) and the hydrophilic donor phase, $\mathrm{h}_{\mathrm{T}}$ is the length of the transcellular route and $D_{T}$ is the diffusion coefficient in the lipophilic phase. The epidermis and gastrointestinal epithelium are regarded primarily as a lipoidal barrier. Unlike the paracellular transport which utilizes the limited area of the intercellular space, transcellular transport has a large surface area available and the diffusion path length is relatively short, resulting in a generally high permeability of lipophilic compounds with a high partition coefficient. When the above mentioned simple diffusion models extend to dynamic processes, the diffusant will travel through the route which offers the least resistance, indicating that drug diffusion across the mucosal membrane may involve a mixture of these two pathways (Zhang and Robinson, 1996).

\section{Dosage Forms for Drug Delivery in the Oral Cavity}

Although the oral mucosa offers the opportunity to use various controlled release delivery systems as well as conventional dosage forms, it has been recognized as having certain drawbacks such as the accidental swallowing of the dosage 
form itself, relatively short retention time and abrasion or erosion caused by the movement of the mouth, especially, the tongue when speaking and masticating and the loss of the drug by salivary wash-out. As mucoadhesive materials are developed, numerous attempts have been made to address the problems associated with retention time of the delivery system and prevention of continuous dilution of the drug by salivary flow. Table I shows the bioadhesive materials used to formulate mucoadhesive oral mucosal delivery systems. Polyacrylic acid $\left(\mathrm{Carbopol}^{\circledR}\right)$ and cellulose derivatives (HPC, HEC and $\mathrm{NaCMC}$ ) have been widely used due to excellent mucoadhesiveness and their biocompatible nature. Kellaway and Warren (1996) have extensively reviewed the use of hydrogels formulated by mucoadhesive polymers to achieve the systemic delivery of drugs via the buccal mucosa. There are a variety of dosage forms mainly prepared with bioadhesive polymers for oral mucosal drug delivery and the essential features were digested.

\section{Adhesive tablets}

Conventional and adhesive tablets have been used for buccal or sublingual administration as they can easily be prepared, are easy to handle and to remove from the site of administration.
These tablets are typically prepared by directly compressing the active substance and suitable pharmaceutical excipients or a granulated mixture of active and inactive ingredients. When peptide or protein drugs are the active substance, the direct compression method would be the most appropriate as it does not essentially involve the use of water or organic solvent and heat (i.e. no granulation and drying process), which might cause the deactivation of the peptide or proteins. Conventional tablets are mainly chosen when the prompt effect of a drug is required, for instance nitroglycerin. In contrast, the adhesive tablets have been used for the purpose of improving administration time, achieving controlled release, preventing the loss of a drug fraction and continuous dilution by salivary wash-out and allowing drinking and speaking (Merkle et al., 1990). Since, to prepare adhesive tablets, mucoadhesive materials are added, the incorporated drugs are released generally for a prolonged period of time. It is clear that to reach the initial therapeutic plasma level of a drug is difficult by such mucoadhesive devices. Lee and Chien (1995) have designed the bilayer mucoadhesive polymer system consisting of fast-release layer and sustained-release layer. LHRH is encapsulated in both layers to achieve prompt absorption by the fast-release layer and to maintain therapeutic level by the sustained-release layer.

Table I. Mucoadhesive Materials Used for Oral Mucosal Delivery Systems

\begin{tabular}{|c|c|c|c|c|}
\hline Materials & Dosage form & Drugs & $\begin{array}{l}\text { Site of } \\
\text { application }\end{array}$ & References \\
\hline HPC-H + Carbopol 934P & Core tablet & Lidocaine & Gingiva & Nagai and Kornish (1987) \\
\hline HEC, HPC, PVP, PVA & Laminated patch & TRH & Buccal & Anders and Merkle (1989) \\
\hline Poly(acrylic acid) & Patch & Oxytocin & Buccal & Kellaway and Warren (1991) \\
\hline Drum-dried waxy maize + PEG 750 & Tablet & Sodium fluoride & Oral & Bottenberg et al. (1991) \\
\hline Polyetherurethane & Disc & $\begin{array}{l}\text { Glucose, sucrose, inulin, } \\
\text { heparin }\end{array}$ & Buccal & Yang and Knutson (1992) \\
\hline $\begin{array}{l}\text { Acrylic-grafted starch, } \\
\text { Methylmethacrylic-grafted starch }\end{array}$ & Microsphere & Isosorbide dinitrate & Buccal & Vyas and Jain (1992) \\
\hline $\begin{array}{l}\text { Carbopol 934P }+ \text { polyisobutylene }+ \\
\text { polyisoprene }\end{array}$ & Patch & Buprenorphine & Buccal & Guo (1994) \\
\hline $\begin{array}{l}\text { PVA + polyoxyethylene octylphenyl } \\
\text { ether }\end{array}$ & Gel & Ergotamine tartrate & Buccal & Tsutsumi et al. (1994) \\
\hline Milk protein & Tablet & Morphine & Buccal & Beyssac et al. (1994) \\
\hline Methocel K4M + Carbopol 934P & Two layer tablet & Acitretin & Buccal & Minghetti and Montanari (1995) \\
\hline Carbopol 934P + PVP K-90 & Bilayer tablet & LHRH & Buccal & Lee and Chien (1995) \\
\hline Glyceryl monooleate & Gel & Brilliant blue & Gum & Engstrom et al. (1995) \\
\hline Carbopol 974P + silicone polymer & Patch & Oxytocin & Buccal & Li et al. (1997) \\
\hline Chitosan & Film/tablet (bilayered) & Nifedipine, propranolol & Buccal & Remunan-Lopez et al. (1998) \\
\hline Thiolated Polycarbophil & Tablet & Leu-enkephalin & Buccal & Langoth et al. (2003) \\
\hline Alginate-Mg, Al-silicate & Film & Nicotine & Buccal & Pongjanyakul and Suksri (2009) \\
\hline
\end{tabular}


Nagai and Konishi (1987) have formulated a mucosal adhesive dosage form of lidocaine using HPC and Carbopol 934P. The dosage form is composed of two bases (core and peripheral) and cap layer. The rapid absorption of lidocaine through the oral mucosa was achieved by the lipophilic nature of the lidocaine and the prolonged action of the lidocaine was obtained by the action of the core base consisting of lidocaine and a lyophilised mixture of HPC and Carbopol 934P.

\section{Adhesive gels}

Gel formulations provide for a large absorption area in contact with the oral mucosa. Greater drug absorption may therefore be achieved, which is a distinct advantage over the tablets in terms of oral mucosal or buccal delivery of a drug. Gels are capable of significant prolongation of the residence time when compared to solutions, which may result in improved absorption. The materials used in the mucoadhesive tablets such as poly(acrylic acid) are also used as the gel forming agents. Glyceryl monooleate and sesame oil gel has been developed to deliver metronidazole to a periodontal pocket. The appearance of glyceryl monooleate and sesame oil system at ambient temperature is fluid enough to inject into the periodontal pocket. Once applied, the glyceryl monooleate and sesame oil system takes up water spontaneously to form a viscous liquid crystalline structure. Meanwhile, the drug incorporated into the system is released as it erodes by the action of neutrophil lysosomal enzyme which degrades the glyceryl monooleate into oleic acid and glycerol (Medlicott et al., 1994).

\section{Adhesive ointments}

Adcortyl in Orabase ${ }^{\circledR}$ (triamcinolone acetonide, E.R. Squibb \& Sons Limited, UK) in which $\mathrm{NaCMC}$, pectin and gelatin incorporated into a polyethylene-paraffin base is a representative adhesive ointment. It is generally believed that a lipophilic base is more appropriate than a hydrophilic base for an oral ointment because the aqueous saliva would rapidly disperse the hydrophilic ointment. Ishida et al. (1983) have prepared oral mucosal ointments for the delivery of prednisolone and used Carbopol 934P with one of the following ointment bases i.e. white petrolatum (oleaginous base), hydrophilic petrolatum (w/o type emulsion base) and absorptive ointment (w/ o type emulsion base). They concluded from results obtained from the in vivo absorption test (hamster cheek pouch) that no absorption of prednisolone was detected from the ointments of hydrophilic petrolatum and absorptive ointment bases whereas the absorption of the drug occurred from the white petrolatum base containing Carbopol 934P. Bremecker et al. (1984) have also introduced a mucoadhesive ointment based on neutralized polymethacrylic acid methyl ester to reduce the irritation to the mucous membrane. This ointment exhibited no unpleasant sensation and had good adhesiveness in 18 patients with lichen ruber planus.

\section{Adhesive patches}

Buccal adhesive patches are attached mainly to the center of the buccal mucosa (patches with $<10-15 \mathrm{~cm}^{2}$ in size and ellipsoid shape) or to sublingual and gingival site (patches with $1-2 \mathrm{~cm}^{2}$ in size) for a period generally of less than several hours. A high flexibility and smooth surface of the buccal adhesive patch may contribute to decrease mechanical irritation or local discomfort. The drug release profiles and direction from the patches vary, depending on the absence and presence of an impermeable backing layer or the amount and kind of polymeric materials employed to give mucoadhesiveness and controlled or sustained release. The presence of an impermeable layer surrounding a patch, except for one surface area (Fig. 1A) and patches containing core-type drug reservoir (Fig. 1B) make the drug release unidirectional. The model patches shown in Fig. $1 \mathrm{C}$ and $\mathrm{D}$ will be suitable for the

A

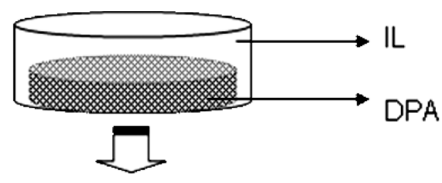

B

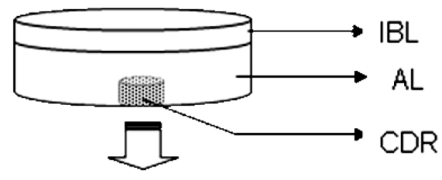

C

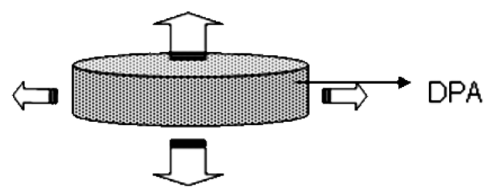

$\mathrm{D}$

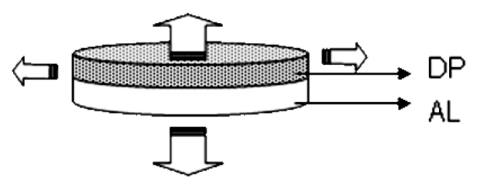

$E$

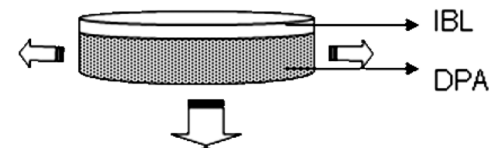

Figure 1. Schematic presentation of possible unidirectional $(A, B)$ and multidirectional $(C, D, E)$ release from the buccal adhesive patches. Key: AL, adhesive layer; CDR, core drug reservoir; IL, impermeable layer; IBL, impermeable backing layer; DP, drug and polymer mixture without adhesives; DPA, drug and polymer mixture with adhesives. 
treatment of intraoral disease, while the patches like Fig. 1E will deliver drugs transmucosally and/or orally (DeGrande et al., 1996).

\section{Chewing gums}

Drugs can be delivered via the oral mucosa locally or systemically by chewing a gum. Chlorhexidine, carbamide and sodium bicarbonate, sulfonamides, fluoride and miconazole were active ingredients in treating a local disease in several studies. The chewing gums were selected for drugs extensively metabolized in the liver such as salicylamide, acetylsalicylic acid, verapamil and nicotine to improve bioavailability. Methadone and ascorbic acid were also studied to give faster painrelief effect than the tablet and safer administration for vitamin $\mathrm{C}$ than the chewing tablet that damages the dental enamel causing erosion of the teeth, respectively. This means of administration is well accepted by both adults and children due to its convenience and favourable taste like sweetness. The drug release from the chewing gum has been influenced by the following factors, i.e. chewing time and rate, the aqueous solubility of the drug, the drug content and the composition of the gum (Rassing, 1996). Sustained release of flavours and sweeteners as well as drugs from the chewing gum could be achieved by reducing particle size of the component (sweetener), forming a drug-ion exchange complex and coating or embedding an agent. Reduction of the particle size of the sweetener, saccharin acid, resulted in decreased released rate. The reason for the prolonged release was that the sweetener was protected from contact with saliva by the gum base, and the effect of this protection was greater than the potential increase in release rate due to the increased surface area of the saccharin acid (Rassing, 1996).

\section{Aerosols and sprays}

Oral sprays are marketed for local and systemic therapy. Coro-Nitro Spray ${ }^{\circledR}$ (glyceryl trinitrate, Boehringer Mannheim UK) and Nitrolingual Spray ${ }^{\circledR}$ (glyceryl trinitrate, Lipha Pharmaceuticals Limited, UK) are metered-dose oromucosal spray for very rapid buccal or sublingual absorption from the spray droplets producing an almost immediate effect. On the other hand, Difflam Spray ${ }^{\circledR}$ (benzydamine hydrochloride, 3M Health Care Limited, UK) and Corsodyl Spray ${ }^{\circledR}$ (chlorhexidine gluconate, GlaxoSmithKline Consumer Healthcare, UK) are intended for local action. Although aerosols and sprays are fascinating for some cases their uses have largely been limited because it is required to co-develop the packaging system propelling drugs and the production cost is expected quite high.

\section{Conclusions}

Various dosage forms have been developed for the delivery of drugs in the oral cavity locally and systemically. Although the oral cavity offers numerous advantages as a site for drug delivery, for improved drug therapy the development of the dosage forms with proper characters is needed, such as an ability to be retained for a desired period of time, to enhance drug permeability for systemic use, and to resist the damage caused by the physiology of the oral cavity. Mucoadhesive biomaterials are thus frequently involved in the formulation of drug delivery devices used in the oral cavity since they provide enhanced retention of the dosage forms and controlled drug delivery.

\section{Acknowledgement}

This study was supported by a grant of the Korean Health Technology R\&D Project, Ministry for Health, Welfare \& Family Affairs, Republic of Korea (A092018).

\section{References}

Anders, R., Merkle, H.P., 1989. Evaluation of laminated mucoadhesive patches for buccal drug delivery. Int. J. Pharm. 49, 231-240.

Beyssac, E., Touaref, F., Aiache, J-M., Sandouk, P., Haguenauer, D., 1994. In vivo evaluation of a bioadhesive controlled release buccal morphine tablet. Proceed. Int. Symp. Control. Rel. Bio. Mater. 21, 553-554.

Bottenberg, P., De Muynck, C., Bouckaert, S., Cleymaet, R., Coomans, D., Slop, D., Remon, J.P., 1991. Development and testing of bioadhesive, fluoride-containing slow release tablets for oral use, Part II: In vivo fluoride release. Proceed. Int. Symp. Control. Rel. Bio. Mater. 18, 636-637.

Bremecker, K-D., Strempel, H., Klein, G., 1984. Novel concept for a mucosal adhesive ointment. J. Pharm. Sci. 73, 548-552.

DeGrande, G., Benes, L., Horriere, F., Karsenty, H., Lacoste, C., McQuinn, R., Guo, J.-H., Scherrer, R., 1996. Specialized oral mucosal drug delivery systems: Patches. In: Oral Mucosal Drug Delivery, Marcel Dekker, New York.

de Vries, M.E., Bodde, H.E., Verhoef, J.C., Junginger, H.E., 1991. Developments in buccal drug delivery. Crit. Rev. Ther. Drug Carr. Syst. 8, 271-303.

Engstrom, S., Ljusberg-Wahren, H., Gustafsson, A., (1995). Bioadhesive properties of the monoolein-water system. Pharm. Tech. Eur. 7, 14-17.

Gandhi, R.B., Robinson, J.R., 1994. Oral cavity as a site for bioadhesive drug delivery. Adv. Drug Del. Rev. 13, 43-74.

Gou, J-H., 1994. Bioadhesive polymer buccal patches for buprenorphine controlled delivery: Formulation, in-vitro adhe- 
sion and release properties. Drug Dev. Ind. Pharm. 20, 2809 2821.

Ishida, M., Nambu, N., Nagai, T., 1983. Ointment-type oral mucosal dosage form of Carbopol containing prednisolone for treatment of aphtha. Chem. Pharm. Bull. 31, 1010-1014.

Karsenty, H., Chau, I., Lacoste, C., Benes, L., Horriere, F., Degrande, G., 1994. Safety profile and patient acceptance of an original transmucosal delivery system. Proceed. Int. Symp. Control. Rel. Bio. Mater. 21, 549-550.

Kellaway, I.W., Warren, S.J., 1991. Mucoadhesive hydrogels. Proceed. Int. Symp. Control. Rel. Bio. Mater. 18, 73-74.

Langoth, N., Kalbe, J., Bernkop-Schnurch, A., 2003. Development of buccal drug delivery systems based on a thiolated polymer. Int. J. Pharm. 252, 141-148.

Lee, Y., Chien, Y.W., 1995. Oral mucosa controlled delivery of LHRH by bilayer mucoadhesive polymer systems. J. Control. Release 37, 251-261.

Li, C., Bhatt, P.P., Johnston, T.P., 1997. Transmucosal delivery of oxytocin to rabbits using a mucoadhesive buccal patch. Pharm. Dev. Tech. 2, 265-274.

Medlicott, N.J., Rathbone, M.J., Tucker, I.G., Holborow, D.W., 1994. Delivery systems for the administration of drugs to the periodontal pocket. Adv. Drug Del. Rev. 13, 181-203.

Merkle, H.P., Anders, R., Wermerskirchen, A., Raehs, S., Wolany, G., 1990. Buccal routes of peptide and protein drug delivery. In: Peptide and Protein Drug Delivery, Marcel Dekker, New York.

Minghetti, P., Montanari, L., 1995. Buccoadhesive two-layer tablet for topical delivery of acitretin. Proceed. 1st World Meeting of APGI/APV, Budapest, 753-754.

Nagai, T., Konishi, R., 1987. Buccal/gingival drug delivery systems. J. Control. Release 6, 353-360.

Nantwi, P.K.K., Cook, D.J., Rogers, D.J., Smart, J.D., 1997. Lectins for drug delivery within the oral cavity - Investigation of lectin binding to oral mucosa. J. Drug Target. 5, 45-55.

Pongjanyakul, T., Suksri, H., 2009. Alginate-magnesium aluminium silicate films for buccal delivery of nicotine. Coll. Surf. B: Biointerf. 74, 103-113.

Rassing, M.R., 1996. Specialized oral mucosal drug delivery systems: Chewing gum. In: Oral Mucosal Drug Delivery, Marcel Dekker, New York.

Rathbone, M.J., Drummond, B.K., Tucker, I.G., 1994. The oral cavity as a site for systemic drug delivery. Adv. Drug Del. Rev. $13,1-22$.

Remunan-Lopez, C., Portero, A., Vila-Jato, J.L., Alonso, M.J., 1998. Design and evaluation of chitosan/ethylcellulose mucoadhesive bilayered devices for buccal drug delivery. J. Control. Release 55, 143-152.

Shojaei, A., Li, X., 1997. Determination of transport route of acyclovir across buccal mucosa. Proceed. Int. Symp. Control. Rel. Bio. Mater. 24, 427-428.

Tsutsumi, K., Takayama, K., Machida, Y., Ebert, C.D., Nakatomi, I., Nagai, T., 1994. Formulation of buccal mucoadhesive dosage form of ergotamine tartrate. STP Pharm. Sci. 4, 230-234.

Utoguchi, N., Watanabe, Y., Matsumoto, M., 1996. Carrier-mediated transport of monocarboxylic acids in primary cultured epithelial cells from rabbit oral mucosa. Proceed. Int. Symp. Control. Rel. Bio. Mater. 23, 249-250.

Vyas, S.P., Jain C.P., 1992. Bioadhesive polymer-grafted starch micospheres bearing isosorbide dinitrate for buccal administration. J. Microencap. 9, 457-464.

Yang, B., Knutson, K., 1992. Buccal delivery system for polar solutes. Proceed. Int. Symp. Control. Rel. Bio. Mater. 19, 409410.

Zhang, H., Robinson, J.R., 1996. Routes of drug transport across oral mucosa. In: Oral Mucosal Drug Delivery, Marcel Dekker, New York. 